\title{
THE INTELLIGENT INFRASTRUCTURE FOR E-BUSINESS
}

\author{
(an extended Abstract)
}

\author{
Liba Svobodova \\ IBM Research \\ Zurich Research Laboratory \\ CH-8803 Rüschlikon \\ Switzerland \\ svo@ zurich.ibm.com
}

As e-business continues to gain momentum, it also develops into more complex and more dynamic forms, both in the $\mathrm{B} 2 \mathrm{C}$ (Business to Consumer) sector, and even more so in the B2B (Business to Business) arena. These trends need to be enabled by increased intelligence and capabilities in the network infrastructure and services, which will evolve into an intelligent infrastructure for e-business.

Dynamic e-business will enable very rapid establishment of business partnerships and contracts over the global network, without any prior business relation developed in the traditional way. Online business directories, e-markets, and other kinds of e-intermediaries will be deployed to locate suitable partners who can provide desired products, parts, consulting on a specific issue, or financial investment. Trusted e-intermediaries will also offer services to verify the trustworthiness of potential partners and facilitate the negotiation and closure of a contract. All of this will happen at electronic speed, as these processes will be conducted over the network in a highly automated way. Such dynamic processes will be particularly useful in the creation and provisioning of application-level, fully electronic services, or e-services. The providers need to introduce such services to the market quickly and test their market value and acceptance without heavy investments in their own equipment; this need will be addressed by e-utilities, which will become an inseparable part of the intelligent infrastructure. E-utilities will manage thousands of modular servers and storage units that can be allocated on demand to a specific customer, service, or business process, as well as securely released and reallocated as the demand changes. 
E-utilities themselves will require a very flexible infrastructure that will be able to support an almost instantaneous reallocation of resources. This needs to be supported by sophisticated monitoring of the utilization across these huge server and storage farms and scheduling and optimization based on the immediate application requirements and their specific service level agreements (SLA) as well as on the total demand, resource status, and overall policies. Dynamic pricing of computing and network resources usage will be deployed to control the demand. The Project Oceano [1] is addressing these multifaceted issues of e-utilities.

On the end-user side, e-services have to become much more interactive and personalizable. The proliferation of mobile devices having data communication and processing capabilities is one of the main drivers of new kinds of services. A well-known example of a highly personalized service is monitoring performance of selected stocks - the user should be able to specify in simple terms what information he/she wants and how it should be delivered. The service, supported by the intelligent infrastructure, needs to determine whether and how to notify the subscriber, and based on the device the subscriber is using at the time, choose the appropriate retrieval mode (push or pull), the presentation mode (text, voice), and the format. The research platform Whale [2] is a middleware supporting multichannel applications with these features. As the number of users who wish to receive real-time information in a highly personalized way grows to tens or even hundreds of thousands, an intelligent form of personalized distribution will be needed. Gryphon [3] is a highly scalable and personalizable publish/subscribe middleware aimed at distributing large volumes of data in real time over a global network.

The Internet has already evolved significantly from no more than pipes that deliver data in packets to an infrastructure that provides a multiplicity of services, such as multicast, secure connections, directories, caching, and transcoding. To grow the intelligence in the network requires powerful computing resources - these will be provided at the edge of the core networks by edge servers. The transport network will become increasingly less visible to the end users of e-services and applications. E-business processes will run on top of dynamically established virtual private networks [4]. As the bottom line, this infrastructure has to be extremely dependable. Not only does it have to be able to automatically heal from component failures, it has to withstand targeted attacks from hackers, as well as deal effectively with very sudden shifts in resource availability and demand. Continuous intrusion detection [5] will become a standard function in the integrated system and network management. The intelligent infrastructure for e-business will be in fact a very large and complex distributed system environment, where interoperability requirements will go far beyond the functional aspects. 
The IBM Research Division has many projects addressing the different aspects of the Intelligent Infrastructure. The cited projects [1]-[5] are illustrative examples.

\section{References}

[1] e-utilities - Project Oceano: http://www.research.ibm.com/oceanoproject/

[2] Mobile Computing Platform (Whale): http://www.zurich.ibm.com/csc/mobile/index.html

[3] Gryphon: http://www.research.ibm.com/gryphon/home.html

[4] Dynamic Service Provisioning: http://www.zurich.ibm.com/csc/distribsys/icorp.html

[5] Intrusion Detection: http://www.zurich.ibm.com/csc/infosec/gsal.html 